\title{
Note about canonical description of T-duality along light-like isometry
}

\author{
J. Klusoñ ${ }^{\mathrm{a}}$ \\ Department of Theoretical Physics and Astrophysics, Faculty of Science, Masaryk University, Kotlářská 2, 61137 Brno, Czech Republic
}

Received: 18 December 2019 / Accepted: 27 January 2020 / Published online: 7 February 2020

(C) The Author(s) 2020

\begin{abstract}
In this short note we analyze canonical description of T-duality along light-like isometry. We show that Tduality of relativistic string theory on this background leads to non-relativistic string theory action on T-dual background.
\end{abstract}

\section{Introduction and summary}

It is well known that two string theories, one defined on the background with compact dimension of radius $R$, and the second one defined on background with compact dimension of radius $R^{\prime}=\frac{\alpha^{\prime}}{R}$, are equivalent. This duality has its origin in the extended nature of the string when we can exchange momentum with winding numbers, respectively. The most powerful description of such a duality is given in terms of Buscher's rules [1,2] of the transformations of the background fields under T-duality. More explicitly, we start with string sigma model on the background where the background metric possesses one isometry. Then we gauge this isometry so that this is now local symmetry on the string world-sheet when we introduce corresponding covariant derivative and two dimensional gauge field. Since this gauge field has to be non-dynamical in order not to change physical content of the theory we add to the action term that ensures that the field strength of this gauge field is zero on shell. As the next step we fix the gauge when we take the world-sheet mode that parameterizes direction with gauged isometry to be zero. Then we can solve the flatness of the gauge field by introducing a new scalar mode that parameterizes the string propagating along dual coordinate where now the background fields are related to the original ones through Buscher's rules.

There is an alternative approach to this standard treatment of T-duality which is description of T-duality with the help of canonical transformations $[3,4]$. This procedure is based on the Hamiltonian form of string in the background that possesses an isometry. Then we perform specific canonical

a e-mail: klu@physics.muni.cz transformations of the world-sheet mode and its conjugate momenta that correspond to coordinate along this isometry. As a result we obtain a new Hamiltonian for dual theory. Finally we perform inverse Legendre transformations to Tdual Lagrangian with the background fields again determined by Buscher's rules.

While this procedure is well established for space-like isometry the case of light-like isometry is much less known and analyzed. The aim of this short note is to focus on this problem, inspired by recent analysis of T-duality in the context of non-relativistic string theories [5,6], where nonrelativistic strings were firstly introduced in seminal papers $[7,8] .{ }^{1}$ In fact, as we will show explicitly in the next section, the canonical analysis of T-duality for string with light-like isometry leads to T-dual action where the kinetic term for dual coordinate is missing. Then in order to solve this problem we introduce two auxiliary fields $\lambda^{+}$and $\lambda^{-}$in such a way that the original Lagrangian is quadratic in coordinate that we dualize. However we still have to ensure that solving equations of motion for $\lambda^{+}, \lambda^{-}$we return to the original Lagrangian density. When we have an extended Lagrangian density we can find its Hamiltonian form and then we perform canonical transformation corresponding Tduality along original light-like coordinate. From this T-dual Hamiltonian we derive corresponding Lagrangian density and we find that the background fields transform according to Buscher's rules. The most remarkable fact concerning T-dual Lagrangian density is that it is linear in auxiliary fields $\lambda^{+}, \lambda^{-}$ that is characteristic property of non-relativistic string theory action $[5,7,10]$. To see this in more details we solve the equations of motion for $\lambda^{+}, \lambda^{-}$that imply that Lagrangian density has the form of non-relativistic string with specific induced world-sheet metric. Clearly for the form of background that was studied in $[5,6]$ the non-relativistic string corresponds to string in stringy Newton-Cartan background. We also show

\footnotetext{
${ }^{1}$ For related works, see for example [9-16].
} 
that the relativistic background with given components of the metric and NSNS two form maps to the family of nonrelativistic backgrounds that are related by field redefinitions which is in agreement with the paper [5].

We mean that this result is very interesting since it shows that string theory on the background with light-like isometry is T-dual to specific form of non-relativistic string. This could be very useful for the quantum description of string theory on the background with light-like isometry, following recent interesting paper [10]. It would be also very interesting to extend the canonical analysis of T-duality transformation presented in this paper to the case of Green-Schwarz superstring. We hope to return to this problem in future.

This paper is organized as follows. In the next Sect. 2 we review canonical treatment of T-duality and we show difficulty with its application on the case of string in the background with light-like isometry. Then in Sect. 3 we perform T-duality transformation in case of the extended action and we show that it leads to non-relativistic string in T-dual background.

\section{T-duality in canonical formalism}

In this section we review basic facts about T-duality as a canonical transformations, following $[3,4]$. Let us be more concrete with the definition of the canonical formalism in case of the bosonic string. We have two sets of canonical variables: $p_{\mu}, x^{\mu}$ where $\mu, v=0, \ldots, 25$ and their duals: $\tilde{p}_{\mu}, \tilde{x}^{\mu}$ where we demand that both canonical variables give equivalent equations of motions. In other words we demand that the string action can be written in two equivalent ways

$$
\begin{aligned}
S & =\int d \tau d \sigma\left(p_{\mu} \partial_{\tau} x^{\mu}-\mathcal{H}\right) \\
& =\int d \tau d \sigma\left(\tilde{p}_{\mu} \partial_{\tau} \tilde{x}^{\mu}-\tilde{\mathcal{H}}\right)+\int d \tau \frac{d G}{d \tau}
\end{aligned}
$$

where $G$ is generating function of canonical transformations and where we label world-sheet of the string with two coordinates $\tau$ and $\sigma$. It is well known that there are four possible forms of the generating function that differ in dependence on two sets of canonical variables. In our case we presume that they depend on original and dual variables, in other words $G=G(x, \tilde{x})$. We presume that $G=\int d \sigma \mathcal{G}$ and hence we can write

$$
\begin{aligned}
p_{\mu} \partial_{\tau} x^{\mu}-\mathcal{H}\left(x^{\mu}, p_{\mu}\right)= & \tilde{p}_{\mu} \partial_{\tau} \tilde{x}^{\mu}-\tilde{\mathcal{H}}\left(\tilde{x}^{\mu}, \tilde{p}_{\mu}\right)+\frac{\partial \mathcal{G}}{\partial \tau} \\
& +\frac{\delta \mathcal{G}}{\delta x^{\mu}} \partial_{\tau} x^{\mu}+\frac{\delta \mathcal{G}}{\delta \tilde{x}^{\mu}} \partial_{\tau} \tilde{x}^{\mu}
\end{aligned}
$$

so that comparing we obtain relation

$$
p_{\mu}=\frac{\delta \mathcal{G}}{\delta x^{\mu}}, \quad \tilde{p}_{\mu}=-\frac{\delta \mathcal{G}}{\delta \tilde{x}^{\mu}},
$$

$\tilde{\mathcal{H}}\left(\tilde{x}^{\mu}, \tilde{p}_{\mu}\right)=\mathcal{H}\left(x^{\mu}, p_{\mu}\right)+\frac{\partial \mathcal{G}}{\partial \tau}$.

This is general form of canonical transformation for bosonic string. In case of T-duality transformation we presume that $\mathcal{G}$ does not explicitly depend on $\tau$. Further, we perform canonical transformation with respect to one coordinate, say $x^{25} \equiv y$ that labels isometry direction. In this case the generating function has the form $[3,4]$

$G(y, \tilde{y})=\frac{T}{2} \int d \sigma\left(\partial_{\sigma} y \tilde{y}-y \partial_{\sigma} \tilde{y}\right)$,

where $T=\frac{1}{2 \pi \alpha^{\prime}}$ is string tension. Let us denote momentum conjugate to $\tilde{y}$ as $p_{\tilde{y}}$. Then from the definition of the canonical transformations we derive following relation between $\tilde{y}$ and $p_{\tilde{y}}$ in the form

$p_{\tilde{y}}=-\frac{\delta G}{\delta \tilde{y}}=-T \partial_{\sigma} y$,

$p_{y}=\frac{\delta G}{\delta y}=-T \partial_{\sigma} \tilde{y}$.

With the help of these relations we obtain dual Hamiltonian when we replace $\partial_{\sigma} y$ with $-\frac{1}{T} p_{\tilde{y}}$ and $p_{y}$ with $-T \partial_{\sigma} y$. Let us now be more explicit and consider Polyakov string action in background with light-like isometry along $y$ direction which means that all background fields do not depend on $y$ explicitly and also that the metric component $G_{y y}$ is absent. Then the Lagrangian has the form

$$
\begin{aligned}
\mathcal{L}= & -\frac{T}{2} \sqrt{-\gamma}\left[\gamma^{\alpha \beta} \partial_{\alpha} x^{i} \partial_{\beta} x^{j} G_{i j}+2 \gamma^{\alpha \beta} \partial_{\alpha} x^{i} G_{i y} \partial_{\beta} y\right] \\
& -\frac{T}{2} \epsilon^{\alpha \beta} B_{\mu \nu} \partial_{\alpha} x^{\mu} \partial_{\beta} x^{\nu},
\end{aligned}
$$

where $\epsilon^{\alpha \beta}=-\epsilon^{\beta \alpha}, \epsilon^{\tau \sigma}=1$ and where $i, j=0, \ldots, 24$. Let us introduce following $1+1$ parameterization of the world-sheet metric $\gamma_{\alpha \beta}$ as

$$
\begin{aligned}
& \gamma_{\alpha \beta}=\left(\begin{array}{cc}
-N^{2}+N^{\sigma} \omega N^{\sigma} & N^{\sigma} \omega \\
N^{\sigma} \omega & \omega
\end{array}\right), \\
& \gamma^{\alpha \beta}=\left(\begin{array}{cc}
-\frac{1}{N^{2}} & \frac{N^{\sigma}}{N^{2}} \\
\frac{N^{\sigma}}{N^{2}} & \frac{1}{\omega}-\frac{N^{\sigma} N^{\sigma}}{N^{2}}
\end{array}\right)
\end{aligned}
$$

so that the Lagrangian has the form

$$
\begin{aligned}
\mathcal{L}= & -\frac{T}{2} N \sqrt{\omega}\left[-\nabla_{n} x^{i} G_{i j} \nabla_{n} x^{j}+\frac{1}{\omega} \partial_{\sigma} x^{i} \partial_{\sigma} x^{j} G_{i j}\right. \\
& \left.-2 \nabla_{n} x^{i} G_{i y} \nabla_{n} y+\frac{2}{\omega} \partial_{\sigma} x^{i} G_{i y} \partial_{\sigma} y\right] \\
& -T B_{\mu \nu} \partial_{\tau} x^{\mu} \partial_{\sigma} x^{\nu}, \quad \nabla_{n} \equiv \frac{1}{N}\left(\partial_{\tau}-N^{\sigma} \partial_{\sigma}\right) .
\end{aligned}
$$

From (8) we obtain conjugate momenta

$$
\begin{aligned}
& p_{i}=T \sqrt{\omega} G_{i j} \nabla_{n} x^{j}+T \sqrt{\omega} G_{i y} \nabla_{n} y-T B_{i \mu} \partial_{\sigma} x^{\mu}, \\
& p_{y}=T \sqrt{\omega} G_{y i} \nabla_{n} x^{i}-T B_{y \mu} \partial_{\sigma} x^{\mu} .
\end{aligned}
$$




$$
p_{N} \approx 0, \quad p_{\omega} \approx 0, \quad p_{N^{\sigma}} \approx 0
$$

where $p_{N}, p_{\omega}, p_{N^{\sigma}}$ are momenta conjugate to $N, \omega$ and $N^{\sigma}$, respectively. Then performing standard analysis we obtain the Hamiltonian density in the form

$$
\begin{aligned}
\mathcal{H}= & \frac{N}{2 T \sqrt{\omega}}\left(\pi_{i} G^{i j} \pi_{j}+\pi_{y} G^{y y} \pi_{y}+2 \pi_{y} G^{y i} \pi_{i}\right. \\
& \left.+T^{2} G_{i j} \partial_{\sigma} x^{i} \partial_{\sigma} x^{j}+2 T^{2} \partial_{\sigma} x^{i} G_{i y} \partial_{\sigma} y\right) \\
& +N^{\sigma}\left(p_{i} \partial_{\sigma} x^{i}+p_{y} \partial_{\sigma} y\right),
\end{aligned}
$$

where $\pi_{\mu}=p_{\mu}+T B_{\mu \nu} \partial_{\sigma} x^{\nu}$ and where the inverse metric $G^{\mu \nu}$ has following form

$$
\begin{aligned}
G^{y y} & =-\frac{1}{G_{y i} \tilde{G}^{i j} G_{j y}}, \quad G^{y j}=\frac{G_{y k} \tilde{G}^{k j}}{G_{y i} G^{i j} G_{j y}}, \\
G^{i j} & =\tilde{G}^{i j}-\frac{G_{y k} \tilde{G}^{k i} G_{y l} \tilde{G}^{l j}}{G_{y i} \tilde{G}^{i j} G_{j y}},
\end{aligned}
$$

where $\tilde{G}^{i j}$ is metric inverse to $G_{i j}$ so that $\tilde{G}^{i k} G_{k l}=\delta_{l}^{i}$. From (10) we also see that it is convenient to introduce $N^{\tau}=\frac{N}{2 T \sqrt{\omega}}$ so that $\omega$ disappears from the Hamiltonian as expected.

Then following previous general discussion we perform T-duality in (10) when

$p_{\tilde{y}}=-T \partial_{\sigma} y, \quad p_{y}=-T \partial_{\sigma} \tilde{y}$

so that

$$
\begin{aligned}
\pi_{y} & =-T \partial_{\sigma} \tilde{y}+T B_{y i} \partial_{\sigma} x^{i}=-T \mathbf{V}, \\
\pi_{i} & =p_{i}+T B_{i j} \partial_{\sigma} x^{j}+T B_{i y} \partial_{\sigma} y=k_{i}-B_{i y} p_{\tilde{y}} .
\end{aligned}
$$

Inserting these results into (10) we obtain T-dual Hamiltonian constraint in the form

$$
\begin{aligned}
\mathcal{H}_{\tau}^{T}= & T^{2} \mathbf{V} G^{y y} \mathbf{V}-2 T\left(k_{i}-B_{i y} p_{\tilde{y}}\right) G^{i y} \mathbf{V} \\
& +\left(k_{i}-B_{i y} p_{\tilde{y}}\right) G^{i j}\left(k_{j}-B_{j y} p_{\tilde{y}}\right)+ \\
& +T^{2} G_{i j} \partial_{\sigma} x^{i} \partial_{\sigma} x^{j}-2 T G_{i y} \partial_{\sigma} x^{i} p_{\tilde{y}}
\end{aligned}
$$

We see that for zero NSNS two form the Hamiltonian constraint is linear in $p_{\tilde{y}}$ which suggests possible difficulties with this theory. This can be also seen even in case of non-zero $B_{\mu \nu}$ when we determine corresponding Lagrangian. In fact, using (14) in the T-dual Hamiltonian $H^{T}=\int d \sigma\left(N^{\tau} \mathcal{H}_{\tau}^{T}+\right.$ $\left.N^{\sigma} \mathcal{H}_{\sigma}^{T}\right)$ where $\mathcal{H}_{\sigma}=p_{i} \partial_{\sigma} x^{i}+p_{\tilde{y}} \partial_{\sigma} \tilde{y}$ we obtain time derivatives of $x^{i}$ and $\tilde{y}$ as

$$
\begin{aligned}
\partial_{\tau} x^{i}=\left\{x^{i}, H^{T}\right\}= & 2 N^{\tau} G^{i j}\left(k_{j}-B_{j y} p_{\tilde{y}}\right) \\
& -2 N^{\tau} T G^{i y} \mathbf{V}+N^{\sigma} \partial_{\sigma} x^{i}, \\
\partial_{\tau} \tilde{y}= & \left\{\tilde{y}, H^{T}\right\}=2 N^{\tau} T B_{i y} G^{i y} \mathbf{V} \\
& -2 N^{\tau} B_{i y} G^{i j}\left(k_{j}-B_{j y} p_{\tilde{y}}\right) \\
& -2 N^{\tau} T G_{i y} \partial_{\sigma} x^{i}+N^{\sigma} \partial_{\sigma} \tilde{y}
\end{aligned}
$$

and hence we find

$$
\begin{aligned}
Y & =-B_{i \tilde{y}} X^{i}-2 N^{\tau} T G_{i \tilde{y}} \partial_{\sigma} x^{i}, \quad Y=\partial_{\tau} \tilde{y}-N^{\sigma} \partial_{\sigma} \tilde{y}, \\
X^{i} & =\partial_{\tau} x^{i}-N^{\sigma} \partial_{\sigma} x^{i}
\end{aligned}
$$

which means that there is no relation between $\partial_{\tau} \tilde{y}$ and conjugate momenta. In fact, if we proceed further we obtain naive T-dual Lagrangian density in the form

$$
\begin{aligned}
\mathcal{L}_{\text {naive }}^{T}= & \frac{1}{4 N^{\tau}} X^{i} G_{i j} X^{j}+T G^{i y} \mathbf{V} G_{i j} X^{j} \\
& +N^{\tau} T^{2} G^{i y} \mathbf{V} G_{i j} G^{j y} \mathbf{V}-N^{\tau} T^{2} \mathbf{V} G^{y y} \mathbf{V} \\
& -N^{\tau} T^{2} G_{i j} \partial_{\sigma} x^{i} \partial_{\sigma} x^{j}-T B_{i j} \partial_{\tau} x^{i} \partial_{\sigma} x^{j}
\end{aligned}
$$

and we see that the kinetic term for T-dual variable $\tilde{y}$ is absent which is not satisfactory result. In the next section we will try to resolve this puzzle by introducing two auxiliary fields.

\section{Extended Lagrangian and T-duality}

In order to resolve this puzzle let us introduce an equivalent form of the Lagrangian density to the original one (8). To do this we introduce two auxiliary fields $\lambda^{+}$and $\lambda^{-}$and consider following Lagrangian density

$$
\begin{aligned}
\mathcal{L}= & -\frac{T}{2} N \sqrt{\omega}\left[-\nabla_{n} x^{i} \hat{G}_{i j} \nabla_{n} x^{j}+\frac{1}{\omega} \partial_{\sigma} x^{i} \partial_{\sigma} x^{j} \hat{G}_{i j}\right. \\
& -2 \nabla_{n} x^{i} \hat{G}_{i y} \nabla_{n} y+\frac{2}{\omega} \partial_{\sigma} x^{i} \hat{G}_{i y} \partial_{\sigma} y \\
& -\nabla_{n} y \hat{G}_{y y} \nabla_{n} y+\frac{1}{\omega} \partial_{\sigma} y \hat{G}_{y y} \partial_{\sigma} y+\lambda^{+} \mathbf{A} \\
& \left.+\lambda^{-} \mathbf{B}+\lambda^{+} \lambda^{-}\right]-T \hat{B}_{\mu \nu} \partial_{\tau} x^{\mu} \partial_{\sigma} x^{\nu},
\end{aligned}
$$

where now we have to choose $\mathbf{A}$ and $\mathbf{B}$ in such a way to ensure that $\hat{G}_{\tilde{y} \tilde{y}}=0$ after solving equations of motion for $\lambda^{+}$and $\lambda^{-}$. Explicitly, from (18) we find their equations of motion in the form

$\lambda^{-}=-\mathbf{A}, \quad \lambda^{+}=-\mathbf{B}$.

Inserting these results into (18) we obtain that they give following contribution to the Lagrangian density

$$
-\frac{T}{2} N \sqrt{\omega}\left[\lambda^{+} \mathbf{A}+\lambda^{-} \mathbf{B}+\lambda^{+} \lambda^{-}\right]=\frac{T}{2} \sqrt{\omega} N \mathbf{A B} .
$$

As the next step we presume that $\mathbf{A}$ and $\mathbf{B}$ have the form

$$
\begin{aligned}
& \mathbf{A}=\nabla_{n} x^{i} \mathbf{A}_{i}+\nabla_{n} y \mathbf{Y}^{+}-\frac{1}{\sqrt{\omega}}\left[\partial_{\sigma} x^{i} \mathbf{A}_{i}+\partial_{\sigma} y \mathbf{Y}^{+}\right], \\
& \mathbf{B}=\nabla_{n} x^{i} \mathbf{B}_{i}+\nabla_{n} y \mathbf{Y}^{-}+\frac{1}{\sqrt{\omega}}\left[\partial_{\sigma} x^{i} \mathbf{B}_{i}+\partial_{\sigma} y \mathbf{Y}^{-}\right],
\end{aligned}
$$

so that

$\mathbf{A B}=\nabla_{n} x^{i} \nabla_{n} x^{j} \mathbf{A}_{i} \mathbf{B}_{j}+\nabla_{n} x^{i} \nabla_{n} y\left(\mathbf{A}_{i} \mathbf{Y}^{-}+\mathbf{Y}^{+} \mathbf{B}_{i}\right)$ 


$$
\begin{aligned}
& +\frac{2}{\sqrt{\omega}} \nabla_{n} x^{i} \partial_{\sigma} x^{j}\left(\mathbf{A}_{i} \mathbf{B}_{j}-\mathbf{A}_{i} \mathbf{B}_{j}\right) \\
& +\frac{1}{\sqrt{\omega}} \nabla_{n} x^{i} \partial_{\sigma} y\left(\mathbf{Y}^{-} \mathbf{A}_{i}-\mathbf{Y}^{+} \mathbf{B}_{i}\right)+ \\
& +\nabla_{n} y \nabla_{n} y \mathbf{Y}^{+} \mathbf{Y}^{-}+\frac{1}{\sqrt{\omega}} \nabla_{n} y \partial_{\sigma} x^{i}\left(\mathbf{Y}^{+} \mathbf{B}_{i}-\mathbf{Y}^{-} \mathbf{A}_{i}\right) \\
& -\frac{1}{\omega}\left(\partial_{\sigma} x^{i} \partial_{\sigma} x^{j} \mathbf{A}_{i} \mathbf{B}_{j}+\partial_{\sigma} x^{i} \partial_{\sigma} y\left(\mathbf{A}_{i} \mathbf{Y}^{-}+\mathbf{B}_{i} \mathbf{Y}^{+}\right)\right. \\
& \left.+\partial_{\sigma} y \partial_{\sigma} y \mathbf{Y}^{+} \mathbf{Y}^{-}\right) .
\end{aligned}
$$

For simplicity we begin with our analysis with the minimal case when $\mathbf{A}_{i}=\mathbf{B}_{i}=0$.

\subsection{Minimal case}

First of all we should demand that when we solve the equations of motion for $\lambda^{+}, \lambda^{-}$the Lagrangian (18) with additional contribution given in (22) reduces to the original one with $G_{y y}=0$. This condition implies

$\hat{G}_{y y}+\mathbf{Y}^{+} \mathbf{Y}^{-}=0$

that can be solved as $\mathbf{Y}^{+}=\sqrt{\hat{G}_{y y}}, \mathbf{Y}^{-}=-\sqrt{\hat{G}_{y y}}$. In this case (22) is equal to

$\mathbf{A B}=\left[\nabla_{n} y \nabla_{n} y-\frac{1}{\omega} \partial_{\sigma} y \partial_{\sigma} y\right] \mathbf{Y}^{+} \mathbf{Y}^{-}$.

Inserting this result into (18) and comparing with (8) we obtain following relation between original metric and NSNS two form fields and hatted ones:

$G_{i j}=\hat{G}_{i j}, \quad G_{i y}=\hat{G}_{i y}, \quad B_{\mu \nu}=\hat{B}_{\mu \nu}$.

Now we return to (18) that for $\mathbf{A}_{i}=\mathbf{B}_{i}=0$ has the form

$$
\begin{aligned}
\mathcal{L}= & -\frac{T}{2} N \sqrt{\omega}\left[-\nabla_{n} x^{i} \hat{G}_{i j} \nabla_{n} x^{j}-\nabla_{n} y \hat{G}_{y y} \nabla_{n} y\right. \\
& -2 \nabla_{n} x^{i} \hat{G}_{i y} \nabla_{n} y+\frac{1}{\omega} \partial_{\sigma} x^{i} \partial_{\sigma} x^{j} \hat{G}_{i j} \\
& +\frac{2}{\omega} \partial_{\sigma} x^{i} \hat{G}_{i y} \partial_{\sigma} y+\frac{1}{\omega} \partial_{\sigma} y \hat{G}_{y y} \partial_{\sigma} y \\
& +\lambda^{+}\left(\nabla_{n} y-\frac{1}{\sqrt{\omega}} \partial_{\sigma} y\right) \mathbf{Y}^{+}+\lambda^{-}\left(\nabla_{n} y+\frac{1}{\sqrt{\omega}} \partial_{\sigma} y\right) \mathbf{Y}^{-} \\
& \left.+\lambda^{+} \lambda^{-}\right]-T \hat{B}_{\mu \nu} \partial_{\tau} x^{\mu} \partial_{\sigma} x^{\nu} .
\end{aligned}
$$

As the next step we proceed to the canonical formalism. From Lagrangian density given above we obtain

$$
\begin{aligned}
p_{i}= & T \sqrt{\omega}\left[\hat{G}_{i j} \nabla_{n} x^{j}+\hat{G}_{i y} \nabla_{n} y\right]-T \hat{B}_{i v} \partial_{\sigma} x^{\nu}, \\
p_{y}= & T \sqrt{\omega}\left[\hat{G}_{y y} \nabla_{n} y+\hat{G}_{y i} \nabla_{n} x^{i}-\frac{1}{2} \lambda^{+} \mathbf{Y}^{+}\right. \\
& \left.-\frac{1}{2} \lambda^{-} \mathbf{Y}^{-}\right]-T \hat{B}_{y i} \partial_{\sigma} x^{i} \\
& p_{N} \approx 0, \quad p_{\omega} \approx 0, \quad p_{N^{\sigma}} \approx 0 \quad p_{\lambda^{ \pm}} \approx 0,
\end{aligned}
$$

and hence Hamiltonian density has the form

$$
\begin{aligned}
\mathcal{H}= & \frac{N}{2 \sqrt{\omega} T}\left(\pi_{i} \hat{G}^{i j} \pi_{j}+2 \pi_{i} \hat{G}^{i y}\left(\pi_{y}+\frac{T}{2} \sqrt{\omega} \lambda^{+} \mathbf{Y}^{+}\right.\right. \\
& \left.+\frac{T}{2} \sqrt{\omega} \lambda^{-} \mathbf{Y}^{-}\right)+\left(\pi_{y}+\frac{T}{2} \sqrt{\omega} \lambda^{+} \mathbf{Y}^{+}\right. \\
& \left.+\frac{T}{2} \sqrt{\omega} \lambda^{-} \mathbf{Y}^{-}\right) \hat{G}^{y y}\left(\pi_{y}+\frac{T}{2} \sqrt{\omega} \lambda^{+} \mathbf{Y}^{+}\right. \\
& \left.\left.+\frac{T}{2} \sqrt{\omega} \lambda^{-} \mathbf{Y}^{-}\right)\right)+\frac{T N}{2 \sqrt{\omega}} \partial_{\sigma} x^{i} \hat{G}_{i j} \partial_{\sigma} x^{j} \\
& +\frac{T N}{\sqrt{\omega}} \partial_{\sigma} x^{i} \hat{G}_{i j} \partial_{\sigma} y+\frac{T N}{2 \sqrt{\omega}} \partial_{\sigma} y \hat{G}_{y y} \partial_{\sigma} y \\
& -\frac{T N}{2} \lambda^{+} \partial_{\sigma} y \mathbf{Y}^{+}+\frac{T N}{2} \lambda^{-} \partial_{\sigma} y \mathbf{Y}^{-} \\
& +\frac{T}{2} N \sqrt{\omega} \lambda^{+} \lambda^{-}+N^{\sigma} \mathcal{H}_{\sigma} .
\end{aligned}
$$

Performing rescaling

$\sqrt{\omega} \lambda^{+}=\tilde{\lambda}^{+}, \quad \sqrt{\omega} \lambda^{-}=\tilde{\lambda}^{-}$

and introducing $N^{\tau}=\frac{N}{2 \sqrt{\omega} T}$ we can rewrite the Hamiltonian density into the form

$\mathcal{H}=N^{\tau} \mathcal{H}_{\tau}+N^{\sigma} \mathcal{H}_{\sigma}$,

where

$$
\begin{aligned}
\mathcal{H}_{\tau}= & \pi_{\mu} \hat{G}^{\mu \nu} \pi_{\nu}+T \pi_{\mu} \hat{G}^{\mu y}\left(\tilde{\lambda}^{+} \mathbf{Y}^{+}+\tilde{\lambda}^{-} \mathbf{Y}^{-}\right) \\
& +\frac{T^{2}}{4}\left(\tilde{\lambda}^{+} \mathbf{Y}^{+}+\tilde{\lambda}^{-} \mathbf{Y}^{-}\right) \hat{G}^{y y}\left(\tilde{\lambda}^{+} \mathbf{Y}^{+}+\tilde{\lambda}^{-} \mathbf{Y}^{-}\right) \\
& -T^{2} \tilde{\lambda}^{+} \partial_{\sigma} y \mathbf{Y}^{+}+T^{2} \tilde{\lambda}^{-} \partial_{\sigma} y \mathbf{Y}^{-}+T^{2} \tilde{\lambda}^{+} \tilde{\lambda}^{-} \\
& +T^{2} \partial_{\sigma} x^{\mu} \hat{G}_{\mu \nu} \partial_{\sigma} x^{\nu}, \quad \mathcal{H}_{\sigma}=p_{\mu} \partial_{\sigma} x^{\mu} .
\end{aligned}
$$

Now we are ready to proceed to T-duality transformation that, according to the general discussion presented in previous section, is given by following transformations

$p_{y}=-T \partial_{\sigma} \tilde{y}, \quad p_{\tilde{y}}=-T \partial_{\sigma} y$

so that

$\pi_{y}=-T \partial_{\sigma} \tilde{y}+T \hat{B}_{y i} \partial_{\sigma} x^{i}=-T \mathbf{V}$,

$\pi_{i}=k_{i}-\hat{B}_{i y} p_{\tilde{y}}, \quad k_{i}=p_{i}+T B_{i j} \partial_{\sigma} x^{j}$.

Inserting this result into (31) we obtain T-dual Hamiltonian constraint in the form

$$
\begin{aligned}
\mathcal{H}_{\tau}^{T}= & \mathcal{H}_{\tau}\left(p_{y}=-T \partial_{\sigma} \tilde{y}, \partial_{\sigma} y=-T^{-1} p_{\tilde{y}}\right) \\
= & \left(k_{i}-\hat{B}_{i y} p_{\tilde{y}}\right) \hat{G}^{i j}\left(k_{j}-\hat{B}_{j y} p_{\tilde{y}}\right) \\
& -2 T\left(k_{i}-\hat{B}_{i y} p_{\tilde{y}}\right) \hat{G}^{i y} \mathbf{V}+T^{2} \mathbf{V}^{2} \hat{G}^{y y} \\
& +T\left(k_{i}-\hat{B}_{i y} p_{\tilde{y}}\right) \hat{G}^{i y}\left(\tilde{\lambda}^{+} \mathbf{Y}^{+}+\tilde{\lambda}^{-} \mathbf{Y}^{-}\right) \\
& -T^{2} \mathbf{V} \hat{G}^{y y}\left(\tilde{\lambda}^{+} \mathbf{Y}^{+}+\tilde{\lambda}^{-} \mathbf{Y}^{-}\right) \\
& +\frac{T^{2}}{4}\left(\tilde{\lambda}^{+} \mathbf{Y}^{+}+\tilde{\lambda}^{-} \mathbf{Y}^{-}\right) \hat{G}^{y y}\left(\tilde{\lambda}^{+} \mathbf{Y}^{+}+\tilde{\lambda}^{-} \mathbf{Y}^{-}\right)
\end{aligned}
$$




$$
\begin{aligned}
& +T \tilde{\lambda}^{+} p_{\tilde{y}} \mathbf{Y}^{+}-T \tilde{\lambda}^{-} p_{\tilde{y}} \mathbf{Y}^{-}+T^{2} \tilde{\lambda}^{+} \tilde{\lambda}^{-} \\
& +T^{2} \partial_{\sigma} x^{i} \hat{G}_{i j} \partial_{\sigma} x^{j}-2 T \partial_{\sigma} x^{i} \hat{G}_{i y} p_{\tilde{y}}+p_{\tilde{y}} \hat{G}_{y y} p_{\tilde{y}} .
\end{aligned}
$$

As the next step we derive corresponding Lagrangian density. With the help of Hamiltonian $H^{T}=\int d \sigma\left(N^{\tau} \mathcal{H}_{\tau}^{T}+N^{\sigma} \mathcal{H}_{\sigma}^{T}\right)$ we obtain following equations of motion

$$
\begin{aligned}
X^{i}= & 2 N^{\tau} \hat{G}^{i j}\left(k_{j}-\hat{B}_{j y} p_{\tilde{y}}\right)-2 N^{\tau} T \hat{G}^{i y} \mathbf{V} \\
& +T N^{\tau} \hat{G}^{i y}\left(\tilde{\lambda}^{+} \mathbf{Y}^{+}+\tilde{\lambda}^{-} \mathbf{Y}^{-}\right), \\
Y= & -\hat{B}_{i y} X^{i}+N^{\tau} T\left(\tilde{\lambda}^{+} \mathbf{Y}^{+}-\tilde{\lambda}^{-} \mathbf{Y}^{-}\right) \\
& -2 T N^{\tau} \partial_{\sigma} x^{i} \hat{G}_{i y}+2 N^{\tau} \hat{G}_{y y} p_{\tilde{y}},
\end{aligned}
$$

where

$$
X^{i}=\partial_{\tau} x^{i}-N^{\sigma} \partial_{\sigma} x^{i}, \quad Y=\partial_{\tau} \tilde{y}-N^{\sigma} \partial_{\sigma} \tilde{y} .
$$

Now we see that we can express $p_{\tilde{y}}$ from the last expression as

$$
\begin{aligned}
p_{\tilde{y}}= & \frac{1}{2 N^{\tau} \hat{G}_{y y}}\left(Y+\hat{B}_{i y} X^{i}-N^{\tau} T\left(\tilde{\lambda}^{+} \mathbf{Y}^{+}-\tilde{\lambda}^{-} \mathbf{Y}^{-}\right)\right. \\
& \left.+2 T N^{\tau} \partial_{\sigma} x^{i} \hat{G}_{i y}\right) .
\end{aligned}
$$

To proceed further we have to introduce metric inverse to $\hat{G}^{i j}$. It is easy to see that it has the form

$\tilde{G}_{i j}=\hat{G}_{i j}-\frac{1}{\hat{G}_{y y}} \hat{G}_{i y} \hat{G}_{y j}$

that obeys

$\tilde{G}_{i j} \hat{G}^{j k}=\delta_{i}^{k}, \quad \tilde{G}_{i j} \hat{G}^{j y}=-\frac{\hat{G}_{i y}}{\hat{G}_{y y}}$.

Then we find

$$
\begin{aligned}
\left(k_{i}-\hat{B}_{i y} p_{\tilde{y}}\right)= & \frac{1}{2 N^{\tau}} \tilde{G}_{i j}\left(X^{j}+2 N^{\tau} T \hat{G}^{j y} \mathbf{V}\right. \\
& \left.-T \hat{G}^{j y} N^{\tau}\left(\tilde{\lambda}^{+} \mathbf{Y}^{+}+\tilde{\lambda}^{-} \mathbf{Y}^{-}\right)\right)
\end{aligned}
$$

and hence after some calculations we obtain T-dual Lagrangian density in the form

$$
\begin{aligned}
\mathcal{L}^{T}= & p_{i} \partial_{\tau} x^{i}+p_{\tilde{y}} \partial_{\tau} \tilde{y}-N^{\tau} \mathcal{H}_{\tau}^{T}-N^{\sigma} \mathcal{H}_{\sigma}^{T} \\
= & \frac{1}{4 N^{\tau}}\left(g_{\tau \tau}^{\prime}-2 N^{\sigma} g_{\tau \sigma}^{\prime}+\left(N^{\sigma}\right)^{2} g_{\sigma \sigma}^{\prime}\right) \\
& -N^{\tau} T^{2} g_{\sigma \sigma}^{\prime}-T B_{\mu \nu}^{\prime} \partial_{\tau} \tilde{x}^{\mu} \partial_{\sigma} \tilde{x}^{\nu} \\
& +\frac{T}{2} N^{\tau} \tilde{\lambda}^{+}\left(\nabla_{n} x^{i} \frac{\mathbf{Y}^{+}}{\hat{G}_{y y}}\left(\hat{G}_{i y}-\hat{B}_{i y}\right)-\nabla_{n} \tilde{y} \frac{\mathbf{Y}^{+}}{\hat{G}_{y y}}\right. \\
& \left.+2 T \partial_{\sigma} \tilde{y} \frac{\mathbf{Y}^{+}}{\hat{G}_{y y}}-2 T \frac{\mathbf{Y}^{+}}{\hat{G}_{y y}}\left(\hat{G}_{i y}-\hat{B}_{i y}\right) \partial_{\sigma} x^{i}\right) \\
& +\frac{T}{2} N^{\tau} \tilde{\lambda}^{-}\left(\nabla_{n} x^{i} \frac{\mathbf{Y}^{-}}{\hat{G}_{y y}}\left(\hat{G}_{i y}+\hat{B}_{i y}\right)+\nabla_{n} y \frac{\mathbf{Y}^{-}}{\hat{G}_{y y}}\right.
\end{aligned}
$$

$$
\begin{aligned}
& \left.+2 T \partial_{\sigma} \tilde{y} \frac{\mathbf{Y}^{-}}{\hat{G}_{y y}}+2 T \partial_{\sigma} x^{i} \frac{\mathbf{Y}^{-}}{\hat{G}_{y y}}\left(\hat{G}_{i y}+\hat{B}_{i y}\right)\right) \\
& -N^{\tau} T^{2} \tilde{\lambda}^{+} \tilde{\lambda}^{-}\left(1+\frac{1}{\hat{G}_{y y}} \mathbf{Y}^{+} \mathbf{Y}^{-}\right),
\end{aligned}
$$

where

$g_{\alpha \beta}^{\prime}=G_{\mu \nu}^{\prime} \partial_{\alpha} \tilde{x}^{\mu} \partial_{\beta} \tilde{x}^{\nu}, \quad \tilde{x}^{\mu} \equiv\left(x^{i}, \tilde{y}\right)$

and where

$$
\begin{aligned}
& G_{i j}^{\prime}=\hat{G}_{i j}-\frac{1}{\hat{G}_{y y}} \hat{G}_{i y} \hat{G}_{y j}+\frac{1}{\hat{G}_{y y}} \hat{B}_{i y} \hat{B}_{j y}, \\
& G_{i \tilde{y}}^{\prime}=\frac{\hat{B}_{i y}}{\hat{G}_{y y}}, \quad G_{\tilde{y} j}^{\prime}=-\frac{\hat{B}_{y j}}{\hat{G}_{y y}}, \quad G_{\tilde{y} \tilde{y}}^{\prime}=\frac{1}{\hat{G}_{y y}} \\
& B_{i j}^{\prime}=B_{i j}-\frac{\hat{G}_{i y}}{\hat{G}_{y y}} \hat{B}_{y j}-\frac{\hat{B}_{i y}}{\hat{G}_{y y}} \hat{G}_{y j}, \\
& B_{i \tilde{y}}^{\prime}=\frac{\hat{G}_{i y}}{\hat{G}_{y y}}, \quad B_{\tilde{y} j}^{\prime}=-\frac{\hat{G}_{y j}}{\hat{G}_{y y}}
\end{aligned}
$$

that are standard Buscher's rules [1,2]. However the crucial point of the T-dual Lagrangian is an absence of the term proportional to $\tilde{\lambda}^{+} \tilde{\lambda}^{-}$since $\mathbf{Y}^{+} \mathbf{Y}^{-}=-\hat{G}_{y y}$ from definition. In fact, let us introduce more compact notation

$$
\begin{aligned}
\mathbf{A}_{\mu}^{\prime} & =\left(\frac{\mathbf{Y}^{+}}{\hat{G}_{y y}}\left(\hat{G}_{i y}-\hat{B}_{i y}\right),-\frac{\mathbf{Y}^{+}}{\hat{G}_{y y}}\right), \\
\mathbf{B}_{\mu}^{\prime} & =\left(\frac{\mathbf{Y}^{-}}{\hat{G}_{y y}}\left(\hat{G}_{i y}+\hat{B}_{i y}\right), \frac{\mathbf{Y}^{-}}{\hat{G}_{y y}}\right),
\end{aligned}
$$

so that the Lagrangian density can be written as

$$
\begin{aligned}
\mathcal{L}^{T}= & \frac{1}{4 N^{\tau}}\left(g_{\tau \tau}^{\prime}-2 N^{\sigma} g_{\tau \sigma}^{\prime}+\left(N^{\sigma}\right)^{2} g_{\sigma \sigma}^{\prime}\right) \\
& -N^{\tau} T^{2} g_{\sigma \sigma}^{\prime}-T B_{\mu \nu}^{\prime} \partial_{\tau} \tilde{x}^{\mu} \partial_{\sigma} \tilde{x}^{\nu} \\
& +\frac{T}{2} N^{\tau} \tilde{\lambda}^{+}\left(\nabla_{n} \tilde{x}^{\mu} \mathbf{A}_{\mu}^{\prime}-2 T \partial_{\sigma} \tilde{x}^{\mu} \mathbf{A}_{\mu}^{\prime}\right) \\
& +\frac{T}{2} N^{\tau} \tilde{\lambda}^{-}\left(\nabla_{n} \tilde{x}^{\mu} \mathbf{B}_{\mu}^{\prime}+2 T \partial_{\sigma} \tilde{x}^{\mu} \mathbf{B}_{\mu}^{\prime}\right) .
\end{aligned}
$$

Now the equation of motion for $\tilde{\lambda}^{+}, \tilde{\lambda}^{-}$implies

$$
\nabla_{n} \tilde{x}^{\mu} \mathbf{A}_{\mu}^{\prime}=2 T \partial_{\sigma} \tilde{x}^{\mu} \mathbf{A}_{\mu}^{\prime}, \quad \nabla_{n} \tilde{x}^{\mu} \mathbf{B}_{\mu}^{\prime}=-2 T \partial_{\sigma} \tilde{x}^{\mu} \mathbf{B}_{\mu}^{\prime} .
$$

We can solve these equations as follow. We multiply the first equation with $\partial_{\sigma} \tilde{x}^{v} \mathbf{B}_{v}^{\prime}$ and the second one with $\partial_{\sigma} \tilde{x}^{v} \mathbf{A}_{v}^{\prime}$ and sum so that we obtain

$$
N^{\sigma}=\frac{\partial_{\tau} \tilde{x}^{\mu} \mathbf{M}_{\mu \nu} \partial_{\sigma} \tilde{x}^{\nu}}{\partial_{\sigma} \tilde{x}^{\mu} \mathbf{M}_{\mu \nu} \partial_{\sigma} \tilde{x}^{\nu}}, \quad \mathbf{M}_{\mu \nu}=\frac{1}{2}\left(\mathbf{A}_{\mu}^{\prime} \mathbf{B}_{\nu}^{\prime}+\mathbf{B}_{\mu}^{\prime} \mathbf{A}_{\nu}^{\prime}\right),
$$

where explicitly we have

$$
\mathbf{M}_{i j}=-\frac{1}{\hat{G}_{y y}}\left(\hat{G}_{i y} \hat{G}_{j y}-\hat{B}_{i y} \hat{B}_{j y}\right), \quad \mathbf{M}_{i \tilde{y}}=\frac{\hat{B}_{i y}}{\hat{G}_{y y}},
$$




$$
\mathbf{M}_{\tilde{y} \tilde{y}}=\frac{1}{\hat{G}_{y y}} .
$$

Further, if we multiply two equations given in (46) together we obtain

$N^{\tau}=\frac{\sqrt{-\operatorname{det} \mathbf{M}_{\alpha \beta}}}{2 \mathbf{M}_{\sigma \sigma}}, \quad \mathbf{M}_{\alpha \beta}=\partial_{\alpha} \tilde{x}^{\mu} \mathbf{M}_{\mu \nu} \partial_{\beta} \tilde{x}^{\nu}$

and hence final Lagrangian density has the form of nonrelativistic string action [5]

$\mathcal{L}^{T}=-\frac{T}{2} \sqrt{-\operatorname{det} \mathbf{M}} \mathbf{M}^{\alpha \beta} \hat{g}_{\alpha \beta}^{\prime}-T \hat{B}_{\mu \nu}^{\prime} \partial_{\tau} \tilde{x}^{\mu} \partial_{\sigma} \tilde{x}^{v}$,

where $\mathbf{M}^{\alpha \beta}$ is matrix inverse to $\mathbf{M}_{\alpha \beta}$ so that $\mathbf{M}^{\alpha \beta} \mathbf{M}_{\beta \gamma}=$ $\delta_{\beta}^{\alpha}$. This is very interesting result that shows that T-dual of the string in the background with light-like isometry is nonrelativistic string in dual background.

It is instructive to find explicit form of the Lagrangian density (50). Using (48) we find that $\mathbf{M}_{\alpha \beta}$ is equal to

$$
\begin{aligned}
\mathbf{M}_{\alpha \beta} & =\frac{1}{\hat{G}_{y y}}\left[\left(\partial_{\alpha} \tilde{y}+\hat{B}_{\alpha y}\right)\left(\partial_{\beta} \tilde{y}+\hat{B}_{\beta y}\right)-\hat{G}_{\alpha y} \hat{G}_{\beta y}\right], \\
\hat{G}_{\alpha y} & =\partial_{\alpha} x^{i} \hat{G}_{i y}, \quad \hat{B}_{\alpha y}=\partial_{\alpha} x^{i} \hat{B}_{i y}
\end{aligned}
$$

so that

$$
\operatorname{det} \mathbf{M}=-\frac{1}{\hat{G}_{y y}^{2}}\left(\left(\partial_{\tau} \tilde{y}+\hat{B}_{\tau y}\right) \hat{G}_{\sigma y}-\left(\partial_{\sigma} \tilde{y}+\hat{B}_{\sigma y}\right) \hat{G}_{\tau y}\right)^{2}(52)
$$

We further observe that the induced metric $\hat{g}_{\alpha \beta}^{\prime}$ an NSNS two form $\hat{B}_{\tau \sigma}^{\prime}$ are equal to is equal to

$$
\begin{aligned}
& \hat{g}_{\alpha \beta}^{\prime}=\partial_{\alpha} x^{i} G_{i j} \partial_{\beta} x^{j}+\mathbf{M}_{\alpha \beta}, \\
& \hat{B}_{\mu \nu}^{\prime} \partial_{\tau} \tilde{x}^{\mu} \partial_{\sigma} \tilde{x}^{\nu}=B_{i j} \partial_{\tau} \tilde{x}^{i} \partial_{\sigma} \tilde{x}^{j}-\sqrt{-\operatorname{det} \mathbf{M}},
\end{aligned}
$$

where we also used the fact that in the minimal case $\hat{G}_{i j}, \hat{G}_{i y}$ and $\hat{B}_{i j}, \hat{B}_{i y}$ coincide with $G_{i j}, G_{i y}$ and $B_{i j}, B_{i y}$ respectively. With the help of these result we obtain Lagrangian density in the form

$$
\begin{aligned}
\mathcal{L}= & \frac{T}{2\left(\left(\partial_{\tau} \tilde{y}+B_{\tau}\right) G_{\sigma y}-\left(\partial_{\sigma} \tilde{y}+B_{\sigma y}\right) G_{\tau y}\right)} \epsilon^{\alpha \alpha^{\prime}} \epsilon^{\beta \beta^{\prime}} \\
& \times\left[\left(\partial_{\alpha^{\prime}} \tilde{y}+B_{\alpha^{\prime} y}\right)\left(\partial_{\beta^{\prime}} \tilde{y}+B_{\beta^{\prime} y}\right)-G_{\alpha^{\prime} y} G_{\beta^{\prime} y}\right] \\
& \times G_{i j} \partial_{\alpha} \tilde{x}^{i} \partial_{\beta} \tilde{x}^{j}-T B_{i j} \partial_{\tau} \tilde{x}^{i} \partial_{\sigma} \tilde{x}^{j} .
\end{aligned}
$$

We see that resulting non-relativistic Lagrangian does not depend on the auxiliary metric $\hat{G}_{y y}$. Further, this Lagrangian density agrees with the model studied in [12] when we identify $G_{i y}=\tau_{i}, G_{i j}=h_{i j}-m_{i} \tau_{j}-\tau_{j} m_{i}, B_{i y}=0$, where $\tau_{i}, m_{i}$ and $h_{i j}$ define torsional Newton-Cartan background [12]. We mean that this is very nice consistency check.

In the next section we will study this problem for more general form of terms proportional to Lagrange multipliers.
3.2 The case of non-zero $\mathbf{A}_{i}, \mathbf{B}_{i}$

Now we return to the case when $\mathbf{A}_{i}$ and $\mathbf{B}_{i}$ are not equal to zero. Recall that extended Lagrangian density has the form

$$
\begin{aligned}
\mathcal{L}= & -\frac{T}{2} N \sqrt{\omega}\left[-\nabla_{n} x^{i} \hat{G}_{i j} \nabla_{n} x^{j}+\frac{1}{\omega} \partial_{\sigma} x^{i} \partial_{\sigma} x^{j} \hat{G}_{i j}\right. \\
& -2 \nabla_{n} x^{i} \hat{G}_{i y} \nabla_{n} y+\frac{2}{\omega} \partial_{\sigma} x^{i} \hat{G}_{i y} \partial_{\sigma} y \\
& -\nabla_{n} y \hat{G}_{y y} \nabla_{n} y+\frac{1}{\omega} \partial_{\sigma} y \hat{G}_{y y} \partial_{\sigma} y+\lambda^{+} \mathbf{A}+\lambda^{-} \mathbf{B} \\
& \left.+\lambda^{+} \lambda^{-}\right]-T \hat{B}_{\mu \nu} \partial_{\tau} x^{\mu} \partial_{\sigma} x^{\nu},
\end{aligned}
$$

where now we assume that $\mathbf{A}$ and $\mathbf{B}$ have the form

$$
\begin{aligned}
& \mathbf{A}=\nabla_{n} x^{i} \mathbf{A}_{i}+\nabla_{n} y \mathbf{Y}^{+}-\frac{1}{\sqrt{\omega}}\left[\partial_{\sigma} x^{i} \mathbf{A}_{i}+\partial_{\sigma} y \mathbf{Y}^{+}\right], \\
& \mathbf{B}=\nabla_{n} x^{i} \mathbf{B}_{i}+\nabla_{n} y \mathbf{Y}^{-}+\frac{1}{\sqrt{\omega}}\left[\partial_{\sigma} x^{i} \mathbf{B}_{i}+\partial_{\sigma} y \mathbf{Y}^{-}\right],
\end{aligned}
$$

where $\mathbf{A}_{i}, \mathbf{B}_{i}, \mathbf{Y}^{+}, \mathbf{Y}^{-}$are general space-time fields.

In order to find relation with the original Lagrangian in the background with light-like isometry let us solve the equations of motions $\lambda^{+}$and $\lambda^{-}$and insert the results into (55). Explicitly we find that the resulting contribution is equal to

$$
\begin{aligned}
\frac{T}{2} N \sqrt{\omega} \mathbf{A B}= & \frac{T}{2} N \sqrt{\omega}\left[\nabla_{n} x^{i} \nabla_{n} x^{j} \mathbf{A}_{i} \mathbf{B}_{j}\right. \\
& +\nabla_{n} x^{i} \nabla_{n} y\left(\mathbf{A}_{i} \mathbf{Y}^{-}+\mathbf{Y}^{+} \mathbf{B}_{i}\right) \\
& +\frac{1}{\sqrt{\omega}} \nabla_{n} x^{i} \partial_{\sigma} x^{j}\left(\mathbf{A}_{i} \mathbf{B}_{j}-\mathbf{A}_{j} \mathbf{B}_{i}\right) \\
& +\frac{1}{\sqrt{\omega}} \nabla_{n} x^{i} \partial_{\sigma} y\left(\mathbf{Y}^{-} \mathbf{A}_{i}-\mathbf{Y}^{+} \mathbf{B}_{i}\right) \\
& +\nabla_{n} y \nabla_{n} y \mathbf{Y}^{+} \mathbf{Y}^{-}+\frac{1}{\sqrt{\omega}} \nabla_{n} y \partial_{\sigma} x^{i} \\
& \times\left(\mathbf{Y}^{+} \mathbf{B}_{i}-\mathbf{Y}^{-} \mathbf{A}_{i}\right) \\
& -\frac{1}{\omega}\left(\partial_{\sigma} x^{i} \partial_{\sigma} x^{j} \mathbf{A}_{i} \mathbf{B}_{j}+\partial_{\sigma} x^{i} \partial_{\sigma} y\right. \\
& \left.\left.\times\left(\mathbf{A}_{i} \mathbf{Y}^{-}+\mathbf{B}_{i} \mathbf{Y}^{+}\right)+\partial_{\sigma} y \partial_{\sigma} y \mathbf{Y}^{+} \mathbf{Y}^{-}\right)\right] .
\end{aligned}
$$

Since we demand that extended Lagrangian density describes string with the light-like isometry after solving equations of motion for $\lambda^{+}, \lambda^{-}$we have to demand that

$\hat{G}_{y y}+\mathbf{Y}^{+} \mathbf{Y}^{-}=0$

that can be again solved as

$\mathbf{Y}^{+}=\sqrt{\hat{G}_{y y}}, \quad \mathbf{Y}^{-}=-\sqrt{\hat{G}_{y y}}$.

It is important to stress that remaining fields $\mathbf{A}_{i}, \mathbf{B}_{i}$ appear in the relations between original space-time fields and hatted 
ones that can be easily derived when we insert (57) into (55) and use (59)

$$
\begin{aligned}
\hat{G}_{i j} & =G_{i j}-\frac{1}{2}\left(\mathbf{A}_{i} \mathbf{B}_{j}+\mathbf{A}_{j} \mathbf{B}_{i}\right), \\
G_{i j} & =\hat{G}_{i y}-\frac{1}{2}\left(\mathbf{A}_{i}-\mathbf{B}_{i}\right) \sqrt{\hat{G}_{y y}}, \\
B_{i j} & =\hat{B}_{i j}-\frac{1}{2}\left(\mathbf{A}_{i} \mathbf{B}_{j}-\mathbf{A}_{j} \mathbf{B}_{i}\right), \\
B_{i y} & =\hat{B}_{i y}+\frac{1}{2} \sqrt{\hat{G}_{y y}}\left(\mathbf{A}_{i}+\mathbf{B}_{i}\right) .
\end{aligned}
$$

For further purposes we introduce notation

$$
\begin{aligned}
& \mathbf{A}=\nabla_{n} x^{\mu} \mathbf{A}_{\mu}-\frac{1}{\sqrt{\omega}} \partial_{\sigma} x^{\mu} \mathbf{A}_{\mu}, \quad \mathbf{A}_{\mu}=\left(\mathbf{A}_{i}, \mathbf{Y}^{+}\right), \\
& \mathbf{B}=\nabla_{n} x^{\mu} \mathbf{B}_{\mu}+\frac{1}{\sqrt{\omega}} \partial_{\sigma} x^{\mu} \mathbf{B}_{\mu}, \\
& \mathbf{B}_{\mu}=\left(\mathbf{B}_{i}, \mathbf{Y}^{-}\right), \quad x^{\mu}=\left(x^{i}, y\right) .
\end{aligned}
$$

Now we are ready to proceed to the Hamiltonian formulation of the Lagrangian density (55) when we have following conjugate momenta

$$
\begin{aligned}
p_{\mu}= & T \sqrt{\omega} \hat{G}_{\mu \nu} \nabla_{n} x^{\nu}-\frac{T}{2} \sqrt{\omega}\left(\lambda^{+} \mathbf{A}_{\mu}+\lambda^{-} \mathbf{B}_{\mu}\right) \\
& -T \hat{B}_{\mu \nu} \partial_{\sigma} x^{\nu}, \\
p_{N} \approx & 0, \quad p_{N^{\sigma}} \approx 0, \quad p_{\lambda^{+}} \approx 0, \quad p_{\lambda^{-}} \approx 0 .
\end{aligned}
$$

Following the same analysis as in previous section we obtain Hamiltonian density in the form

$\mathcal{H}=N^{\tau} \mathcal{H}_{\tau}+N^{\sigma} \mathcal{H}_{\sigma}$,

where

$$
\begin{aligned}
\mathcal{H}_{\tau}= & \pi_{\mu} \hat{G}^{\mu \nu} \pi_{v}+T^{2} \partial_{\sigma} x^{\mu} \hat{G}_{\mu \nu} \partial_{\sigma} x^{v} \\
& +T \pi_{\mu} \hat{G}^{\mu v}\left(\tilde{\lambda}^{+} \mathbf{A}_{v}+\tilde{\lambda}^{-} \mathbf{B}_{v}\right) \\
& +T^{2} \partial_{\sigma} x^{\mu}\left(\tilde{\lambda}^{-} \mathbf{B}_{\mu}-\tilde{\lambda}^{+} \mathbf{A}_{\mu}\right)+\frac{T^{2}}{4}\left(\tilde{\lambda}^{+} \mathbf{A}_{\mu}+\tilde{\lambda}^{-} \mathbf{A}_{\mu}\right) \\
& \hat{G}^{\mu \nu}\left(\tilde{\lambda}^{+} \mathbf{A}_{v}+\tilde{\lambda}^{-} \mathbf{B}_{v}\right)+T^{2} \tilde{\lambda}^{+} \tilde{\lambda}^{-}
\end{aligned}
$$

Performing the same T-duality transformation as in previous section we obtain T-dual Hamiltonian

$$
\begin{aligned}
& \mathcal{H}^{T}=N^{\tau} \mathcal{H}_{\tau}^{T}+N^{\sigma} \mathcal{H}_{\sigma}^{T}, \quad \mathcal{H}_{\sigma}^{T}=p_{i} \partial_{\sigma} x^{i}+p_{\tilde{y}} \partial_{\sigma} \tilde{y}, \\
& \mathcal{H}_{\tau}^{T}=\left(k_{i}-\hat{B}_{i y} p_{\tilde{y}}\right) \hat{G}^{i j}\left(k_{j}-\hat{B}_{j y} p_{\tilde{y}}\right) \\
& \quad-2 T \mathbf{V} \hat{G}^{y i}\left(k_{i}-\hat{B}_{i y} p_{\tilde{y}}\right)+T^{2} \mathbf{V}^{2} \hat{G}^{y y} \\
& \quad+T\left(k_{i}-\hat{B}_{i y} p_{\tilde{y}}\right) \hat{G}^{i v}\left(\tilde{\lambda}^{+} \mathbf{A}_{v}+\tilde{\lambda}^{-} \mathbf{B}_{v}\right) \\
& \quad-T^{2} \mathbf{V} \hat{G}^{y v}\left(\tilde{\lambda}^{+} \mathbf{A}_{v}+\tilde{\lambda}^{-} \mathbf{B}_{v}\right) \\
& \quad+T^{2} \partial_{\sigma} x^{i}\left(\tilde{\lambda}^{-} \mathbf{B}_{i}-\tilde{\lambda}^{+} \mathbf{A}_{i}\right) \\
& \quad-T p_{\tilde{y}}\left(\tilde{\lambda}^{-} \mathbf{B}_{y}-\tilde{\lambda}^{+} \mathbf{A}_{y}\right)
\end{aligned}
$$

$$
\begin{aligned}
& +T^{2} \partial_{\sigma} x^{i} \hat{G}_{i j} \partial_{\sigma} x^{j}-2 T p_{\tilde{y}} \hat{G}_{y i} \partial_{\sigma} x^{i}+p_{\tilde{y}}^{2} \hat{G}_{y y} \\
& +\frac{T^{2}}{4}\left(\tilde{\lambda}^{+} \mathbf{A}_{\mu}+\tilde{\lambda}^{-} \mathbf{B}_{\mu}\right) \hat{G}^{\mu \nu}\left(\tilde{\lambda}^{+} \mathbf{A}_{v}+\tilde{\lambda}^{-} \mathbf{B}_{v}\right) \\
& +T^{2} \tilde{\lambda}^{+} \tilde{\lambda}^{-}, \quad p_{\omega} \approx 0 .
\end{aligned}
$$

As the next step we again determine corresponding Lagrangian density using equations of motion for $x^{i}, \tilde{y}$. Since the analysis is completely the same as in previous section we write the final result

$$
\begin{aligned}
\mathcal{L}^{T}= & \frac{1}{4 N^{\tau}}\left(g_{\tau \tau}^{\prime}-2 N^{\sigma} g_{\tau \sigma}^{\prime}+\left(N^{\sigma}\right)^{2} g_{\sigma \sigma}^{\prime}\right)-N^{\tau} T^{2} g_{\sigma \sigma}^{\prime} \\
& -T \hat{B}_{\mu \nu} \partial_{\tau} \tilde{x}^{\mu} \partial_{\sigma} \tilde{x}^{\nu} \\
& +\frac{T}{2} N \tilde{\lambda}^{+}\left(\nabla_{n} \tilde{x}^{\mu} \mathbf{A}_{\mu}^{\prime}-2 T \partial_{\sigma} \tilde{x}^{\mu} \mathbf{A}_{\mu}^{\prime}\right) \\
& +\frac{T}{2} N \tilde{\lambda}^{-}\left(\nabla_{n} \tilde{x}^{\mu} \mathbf{B}_{\mu}^{\prime}+2 T \partial_{\sigma} \tilde{x}^{\mu} \mathbf{B}_{\mu}^{\prime}\right)
\end{aligned}
$$

where

$$
\begin{aligned}
\mathbf{A}_{\mu}^{\prime} & =\left(\left(\frac{\hat{G}_{i y}}{\hat{G}_{y y}} \mathbf{A}_{y}-\frac{\hat{B}_{i y}}{\hat{G}_{y y}} \mathbf{A}_{y}-\mathbf{A}_{i}\right),-\frac{\mathbf{A}_{y}}{\hat{G}_{y y}}\right) \\
& =\frac{1}{\sqrt{\hat{G}_{y y}}}\left(\left(G_{i y}-B_{i y}\right),-1\right), \\
\mathbf{B}_{\mu}^{\prime} & =\left(\left(\frac{\hat{G}_{i y}}{\hat{G}_{y y}} \mathbf{B}_{y}+\frac{\hat{B}_{i y}}{\hat{G}_{y y}} \mathbf{B}_{y}-\mathbf{B}_{i}\right), \frac{\mathbf{B}_{y}}{\hat{G}_{y y}}\right) \\
& =-\frac{1}{\sqrt{\hat{G}_{y y}}}\left(\left(G_{i y}+B_{i y}\right), 1\right),
\end{aligned}
$$

and where $g_{\mu \nu}^{\prime}$ was given in (42) with the background fields given in (43). Note that $\mathbf{A}_{\mu}^{\prime}, \mathbf{B}_{\mu}^{\prime}$ given in (67) agree with (44). On the other when we use (60) together with (43) we obtain following form of the metric $G_{\mu \nu}^{\prime}$ and $B_{\mu \nu}^{\prime}$

$$
\begin{aligned}
G_{i j}^{\prime}= & G_{i j}-\frac{G_{i y} G_{j y}}{\hat{G}_{y y}}+\frac{B_{i y} B_{j y}}{\hat{G}_{y y}}-\frac{1}{2 \sqrt{\hat{G}_{y y}}} G_{i y}\left(\mathbf{A}_{j}-\mathbf{B}_{j}\right) \\
& -\frac{1}{2 \sqrt{\hat{G}_{y y}}}\left(\mathbf{A}_{i}-\mathbf{B}_{j}\right) G_{j y}-\frac{1}{2 \sqrt{\hat{G}_{y y}}}\left(\mathbf{A}_{i}+\mathbf{B}_{i}\right) B_{j y} \\
& -\frac{1}{2 \sqrt{\hat{G}_{y y}}} \mathbf{B}_{i y}\left(\mathbf{A}_{j}+\mathbf{B}_{j}\right), \\
B_{i j}= & B_{i j}-\frac{G_{i y}}{\hat{G}_{y y}} B_{y j}-\frac{B_{i y}}{\hat{G}_{y y}} G_{y j}+\frac{1}{2 \sqrt{\hat{G}_{y y}}}\left(\mathbf{A}_{i}-\mathbf{B}_{i}\right) B_{i j} \\
& -\frac{1}{2 \sqrt{\hat{G}_{y y}}} B_{i y}\left(\mathbf{A}_{j}-\mathbf{B}_{j}+\frac{1}{2 \sqrt{\hat{G}_{y y}}}\left(\mathbf{A}_{i}+\mathbf{B}_{i}\right) G_{j y}\right. \\
& -\frac{1}{2 \sqrt{\hat{G}_{y y}}} G_{i y}\left(\mathbf{A}_{j}+\mathbf{B}_{j}\right),
\end{aligned}
$$




$$
\begin{aligned}
G_{i y}^{\prime} & =\frac{B_{i y}}{\hat{G}_{y y}}-\frac{1}{2 \sqrt{\hat{G}_{y y}}}\left(\mathbf{A}_{i}+\mathbf{B}_{i}\right), \\
B_{i y}^{\prime} & =\frac{G_{i y}}{\hat{G}_{y y}}+\frac{1}{2 \sqrt{\hat{G}_{y y}}}\left(\mathbf{A}_{i}-\mathbf{B}_{i}\right) .
\end{aligned}
$$

We see that relativistic background with light-like isometry that is defined by the metric $G_{\mu \nu}$ and NSNS two form $B_{\mu \nu}$ is mapped to the family of non-relativistic background fields $G_{\mu \nu}^{\prime}, B_{\mu \nu}^{\prime}$ that differ by redefinition given by $\mathbf{A}_{i}, \mathbf{B}_{i}$ and $\hat{G}_{y y}$. This result is in agreement with the analysis presented in [5] and it is again nice consistency check of our analysis.

As the final step we solve the equations of motion for $\tilde{\lambda}^{+}, \tilde{\lambda}^{-}$and we obtain final form of the Lagrangian density for T-dual theory

$$
\begin{aligned}
\mathcal{L}^{T} & =-\frac{T}{2} \sqrt{-\operatorname{det} \mathbf{M}} \mathbf{M}^{\alpha \beta} g_{\alpha \beta}^{\prime}-T B_{\mu \nu}^{\prime} \partial_{\tau} \tilde{x}^{\mu} \partial_{\sigma} \tilde{x}^{\nu}, \\
\mathbf{M}_{\alpha \beta} & =\partial_{\alpha} \tilde{x}^{\mu} \mathbf{M}_{\mu \nu} \partial_{\beta} \tilde{x}^{\nu},
\end{aligned}
$$

where

$$
\begin{aligned}
\mathbf{M}_{\alpha \beta} & =\frac{1}{\hat{G}_{y y}}\left[\left(\partial_{\alpha} \tilde{y}+B_{\alpha y}\right)\left(\partial_{\beta} \tilde{y}+B_{\beta y}\right)-G_{\alpha y} G_{\beta y}\right], \\
G_{\alpha y} & =\partial_{\alpha} x^{i} G_{i y}, \quad B_{\alpha y}=\partial_{\alpha} x^{i} B_{i y} .
\end{aligned}
$$

Let us outline our results. We analyzed T-duality of relativistic string along light-like isometry and we argued that T-dual theory has the form of non-relativistic string action on T-dual background.

Data Availability Statement This manuscript has no associated data or the data will not be deposited. [Author's comment: This is a theoretical study and no experimental data has been listed.]

Open Access This article is licensed under a Creative Commons Attribution 4.0 International License, which permits use, sharing, adaptation, distribution and reproduction in any medium or format, as long as you give appropriate credit to the original author(s) and the source, provide a link to the Creative Commons licence, and indicate if changes were made. The images or other third party material in this article are included in the article's Creative Commons licence, unless indicated otherwise in a credit line to the material. If material is not included in the article's Creative Commons licence and your intended use is not permitted by statutory regulation or exceeds the permitted use, you will need to obtain permission directly from the copyright holder. To view a copy of this licence, visit http://creativecomm ons.org/licenses/by/4.0/.

Funded by SCOAP ${ }^{3}$.

\section{References}

1. T.H. Buscher, A symmetry of the string background field equations. Phys. Lett. B 194, 59 (1987). https://doi.org/10.1016/ 0370-2693(87)90769-6

2. T.H. Buscher, Path integral derivation of quantum duality in nonlinear sigma models. Phys. Lett. B 201, 466 (1988). https://doi.org/ 10.1016/0370-2693(88)90602-8
3. E. Alvarez, L. Alvarez-Gaume, Y. Lozano, A canonical approach to duality transformations. Phys. Lett. B 336, 183 (1994). https:// doi.org/10.1016/0370-2693(94)00982-1. arXiv:hep-th/9406206

4. E. Alvarez, L. Alvarez-Gaume, Y. Lozano, An introduction to $\mathrm{T}$ duality in string theory. Nucl. Phys. Proc. Suppl. 41, 1 (1995). https://doi.org/10.1016/0920-5632(95)00429-D. arXiv:hep-th/9410237

5. E. Bergshoeff, J. Gomis, Z. Yan, Nonrelativistic string theory and T-duality. JHEP 1811, 133 (2018). arXiv:1806.06071 [hep-th]

6. J. Klusoň, Note about T-duality of non-relativistic string. arXiv:1811.12658 [hep-th]

7. J. Gomis, H. Ooguri, Nonrelativistic closed string theory. J. Math. Phys. 42, 3127 (2001). https://doi.org/10.1063/1.1372697. arXiv:hep-th/0009181

8. U.H. Danielsson, A. Guijosa, M. Kruczenski, IIA/B, wound and wrapped. JHEP 0010, 020 (2000). https://doi.org/10.1088/ 1126-6708/2000/10/020. arXiv:hep-th/0009182

9. J. Gomis, J. Gomis, K. Kamimura, Non-relativistic superstrings: a new soluble sector of $\operatorname{AdS}(5) \times S^{* * 5}$. JHEP 0512, 024 (2005). https://doi.org/10.1088/1126-6708/2005/12/ 024. arXiv:hep-th/0507036

10. J. Gomis, J. Oh, Z. Yan, Nonrelativistic string theory in background fields. arXiv:1905.07315 [hep-th]

11. R. Andringa, E. Bergshoeff, J. Gomis, M. de Roo, 'Stringy' Newton-Cartan gravity. Class. Quant. Gravit. 29, 235020 (2012). $\quad$ https://doi.org/10.1088/0264-9381/29/23/235020. arXiv: 1206.5176 [hep-th]

12. T. Harmark, J. Hartong, N.A. Obers, Nonrelativistic strings and limits of the AdS/CFT correspondence. Phys. Rev. D 96(8), 086019 (2017). https://doi.org/10.1103/PhysRevD.96. 086019. arXiv:1705.03535 [hep-th]

13. J. Klusoň, Remark about non-relativistic string in Newton-Cartan background and null reduction. JHEP 1805, 041 (2018). https:// doi.org/10.1007/JHEP05(2018)041. arXiv:1803.07336 [hep-th]

14. J. Klusoň, Nonrelativistic string theory sigma model and its canonical formulation. Eur. Phys. J. C 79(2), 108 (2019). https://doi.org/ 10.1140/epjc/s10052-019-6623-9. arXiv:1809.10411 [hep-th]

15. T. Harmark, J. Hartong, L. Menculini, N.A. Obers, Z. Yan, Strings with non-relativistic conformal symmetry and limits of the AdS/CFT correspondence. JHEP 1811, 190 (2018). https://doi.org/ 10.1007/JHEP11(2018)190. arXiv:1810.05560 [hep-th]

16. J. Klusoň, $(m, n)$-string and D1-brane in stringy Newton-Cartan background. JHEP 1904, 163 (2019). https://doi.org/10.1007/ JHEP04(2019)163. arXiv:1901.11292 [hep-th]

17. J. Klusoň, Hamiltonian for a string in a Newton-Cartan background. Phys. Rev. D 98(8), 086010 (2018). https://doi.org/10. 1103/PhysRevD.98.086010. arXiv:1801.10376 [hep-th] 